\section{SPACE-OUT: Graphics programs to study and to simulate space use and movement patterns}

\author{
MARC BEKOFF and C. WIELAND \\ Department of Environmental, Population \\ and Organismic Biologi". University of Colorado \\ Boulder. Colorado 80309 \\ and

\section{W. A. LAVENDER} \\ Colorado Electro-Optics, Boulder, Colorado 80301
}

Recent reviews (Koeppl, Slade, \& Hoffmann, 1975; Michener, 1979; Waser \& Wiley, 1980, and references therein) have stressed the importance of spatial analyses for furthering our understanding of the ways in which space use and movements within an animal's home range (Burt, 1943) or territory may be related to resources such as food and mates, social interaction patterns, and the evolution of behavior. Raw data for analyzing the use of space can be generated from studies employing trapping (Hayne, 1943), radiotelemetry, direct observation, or some combination of these methods. The programs described here should be useful to researchers interested in analyzing individual movements in twodimensional space.

Program Description. SPACE-OUT contains five programs written in Hewlett-Packard (HP) BASIC that are able to handle data input and analyses and to run simulations of animal movements. All programs are flexible and are easily adapted to deal with data stemming from different studies as long as $(\mathrm{x}, \mathrm{y})$ coordinates can be assigned to places in which individuals are located. The user is led through each program by a series of statements and questions to which answers are required before subsequent analysis is possible.

As written, the programs are stored on data cartridges (HP9800 series or Scotch DC100A) and are used on an HP graphics system that includes a digitizer (HP9874A), desk-top computer (HP9854B), and plotter (HP9872B) that can produce figures in four colors.

Data Input. The program, COYOTE (about 400 lines), is used to input digitized data. If the information is available, the animal is identified (age, sex, breeding condition, social status, health, fitted with a radio collar or not), and a date is assigned to each $(x, y)$ location point. A file name is assigned to each animal

Purchase of the Hewlett-Packard computer graphics system was supported by grants from the National Science Foundation (BNS-79-05770) and the Graduate School of the University of Colorado, Boulder. Programming and supplies were partially paid for by a grant from the National Science Foundation (BNS-79-23463) and a John Simon Guggenheim Memorial Foundation Fellowship, both to M. Bekoff. Genevra Metcalf typed the manuscript. and a two-line description can be entered for future reference. Data can be entered in any order; the program orders the data sequentially, beginning with Point 1. When data input is completed, the $(\mathrm{x}, \mathrm{y})$ coordinates and dates of all points, together with the descriptive information, are stored on a tape cartridge.

Data Analysis. Data are analyzed using a package of compatible programs. The major program, 3DPOLY (about 1,500 lines), produces a printout of all raw data, identifies the animal by file name and descriptive statement, calculates distances between successive locations, generates six two-dimensional convex frequencyuse polygons (Figure 1A) on which each point is labeled with either a number or a symbol, draws a threedimensional plot (which can be rotated in space; Fig. ures $1 \mathrm{~B}$ and $\mathrm{C}$ ) on which the $\mathrm{z}$-axis represents the probability of finding the animal at a specific $(x, y)$ coordinate, and produces a table of probability values. Low probability values can be left out to provide a clearer picture (Figure 1C). The activity center [mean $(x, y)$ coordinate] and other calculations from Koeppl et al. (1975) also are printed.

3DPOLY is a flexible program. The scale is changeable so that the user can input data from a variety of location maps. Our standard scale is $1 \mathrm{~km}=1.64 \mathrm{in}$. We use topological maps available from the U.S. Geological Survey. The program also draws an "eye" to indicate the point from which observations are made.

Two other programs are available. The first, SELECT (about 200 lines), allows the user to select specific monthly data from the general data pool for an individual, so that, for example, space use can be studied during different seasons when food resources vary or when breeding or whelping occur.

The second program, MERGE (about 200 lines), permits the user to study age and sex differences in space use and also to combine location data for individuals living in different types of social groups. For example, we have used MERGE to analyze movement patterns of coyotes (Canis latrans) living in packs, as mated pairs, and as solitary residents (Bekoff \& Wells, 1980).

Simulation. Simulation of movement patterns is accomplished using RAND (about 200 lines), which is compatible with 3DPOLY. RAND replaces COYOTE for data input. Instead of digitizing real data, coordinates are assigned randomly by a random-number generator. The user can choose among a number of options, including: (1) the number of points to be simulated (10 to $2,000)$, (2) the length of the sides of the study plot (from $10 \times 10$ to $100 \times 100$ units), (3) the maximum distance ("step distance") that the individual is allowed to travel on each move, (4) the coordinates at which movements begin, (5) whether output [each $(x, y)$ coordinate; Figure 1D] is labeled with numbers or small 


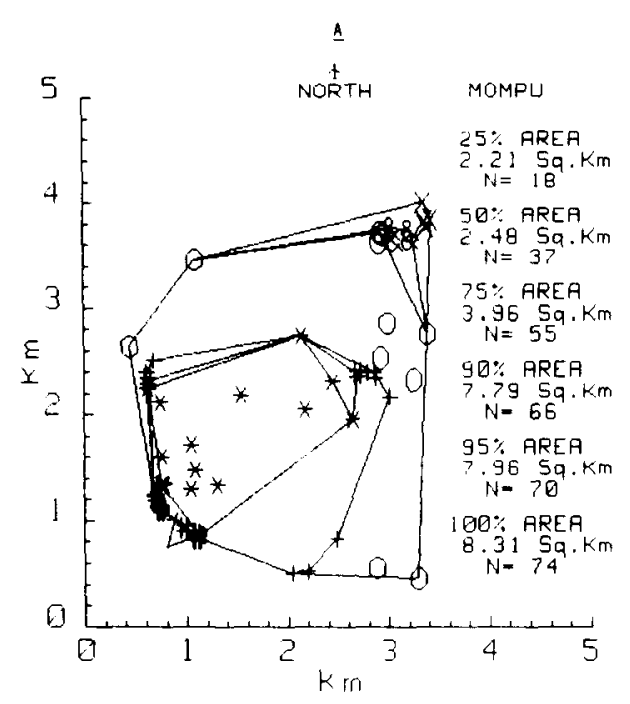

$\underline{c}$

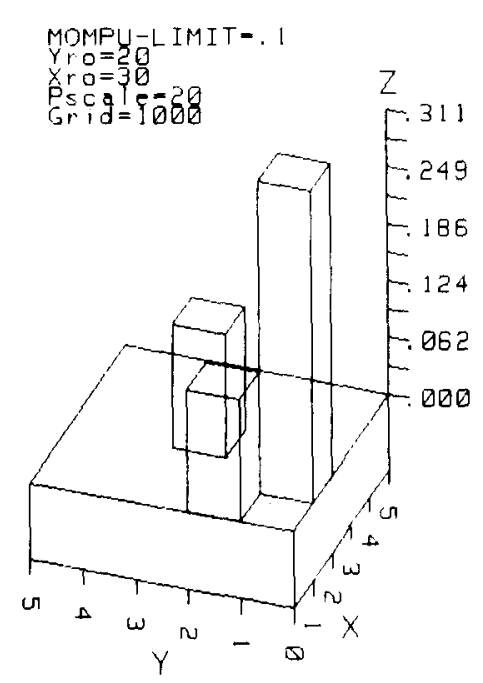

$\underline{B}$

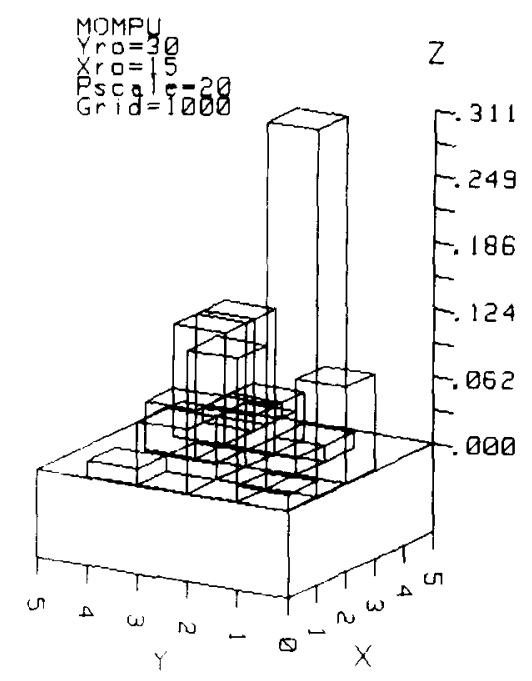

$\underline{\mathbf{D}}$

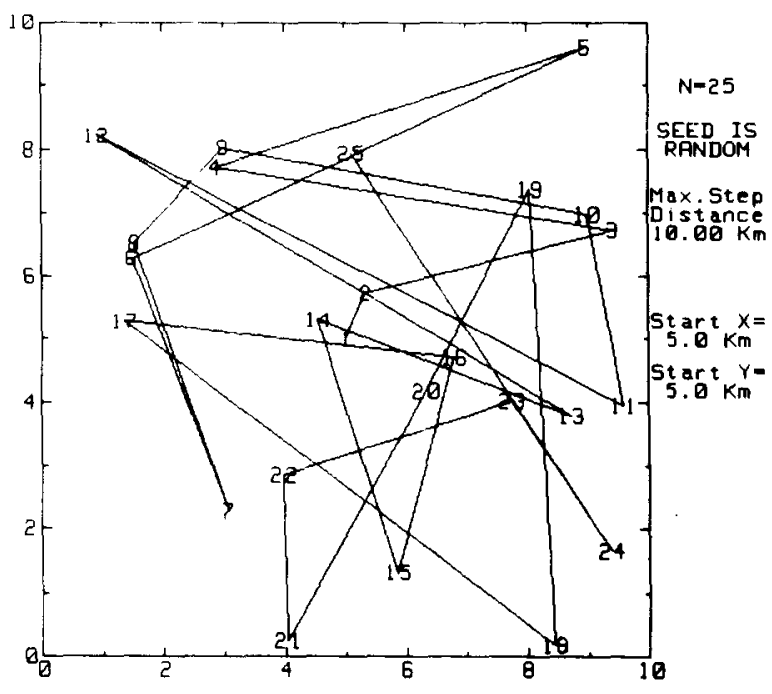

Figure 1. (A) Frequency-use polygons generated by 3DPOLY. Each polygon represents the area encompassed by $25 \%$, $50 \%, 75 \%, 90 \%, 95 \%$, and $100 \%$ of the location points (also see Michener, 1979). In this case, the data are for "MOMPU" (female coyote when her pups were at the den); the $(x, y)$ location of points within each polygon is labeled with a symbol (numbers also can be printed). Area (in square kilometers) and sample size also are printed. The "eye" drawn approximately at $(.8, .8)$ indicates the point from which observations are made. (B) Three-dimensional plot indicating the probability (z-axis) of finding "MOMPU" within 1,000 x 1,000 m grids. Yro and Xro indicate the rotation relative to the $y$-and $x$-axes, Pscale indicates that the actual probabilities have been multiplied by 20 for graphic purposes, and "Grid" represents the length of the sides of the grid (in this case, $1,000 \mathrm{~m}$ ) for which the probabilities are calculated. (C) Three-dimensional plots can be rotated in space (note values for Yro and Xro are different from those in Figure 1B), and low probabilities can be omitted from the display (in this case, LIMIT $=.1$ means that rectangles representing probabilities less than .1 were not drawn). These two options allow for a clearer pictorial representation of data. (D) A simulation of space use and movements using RAND. Twenty-five points (N) were run using a random-number generator to assign $(x, y)$ coordinates. The individual was allowed to move anywhere on the 10 by 10 grid (maximum step distance $=10.00 \mathrm{~km}$ ), and movements began in the center of the area $(5,5)$. Sample size, step distance, starting points, and labeling can be varied. Simulated data can be stored and analyzed using 3DPOLY.

circles, and (6) whether points are connected with straight lines. Individual simulations can be stored on tape and subsequently analyzed using 3DPOLY. We currently are using RAND to determine if there is a relationship between the number of location points and the size of an individual's home range (is there a sample size above which area reaches an asymptote?). We also are comparing the way in which variability in the size of the frequency-use polygons is a function of sample size. RAND also allows a researcher to compare real 
data with simulated data with respect to all information that is produced by $3 \mathrm{DPOLY}$.

Transport to Other Systems. All commands in SPACEOUT dealing with graphical manipulations are specific to the HP system. Also, HP BASIC has built-in matrix operations, and the three-dimensional portions of 3DPOLY rely heavily on this capacity. A minimum of $64 \mathrm{~KB}$ of memory is required. We can supply a description of nonstandard commands specific to HP BASIC to potential users.

The programs are very flexible. When used on a system such as ours, there is no cost for each analysis. Immediate results can be obtained from numerous runs, and hard copies can be made of any and all output.

Program Availability. People interested in receiving program listings should contact the senior author. The only charge is for copying the listings.

\section{REFERENCES}

BeкоF, M., \& Wells, M. C. The social ecology of coyotes. Scientific American, 1980, 242, 130-148.

Bunt, W. H. Territoriality and home range concepts as applied to mammals. Journal of Mammalogy, 1943, 24, 346-352.

HAYNE, D. W. Calculation of size of home range. Journal of Mammalogy, 1943, 30, 1-18.

Koeppl, J. W., Slade, N. A., \& Hoffmann, R. S. A bivariate home range model with possible application to ethological data. Journal of Mammalogy, 1975, 56, 81-90.

MiCHENER, G. R. Spatial relationships and social organization of adult Richardson's ground squirrels. Canadian Journal of Zoology, 1979, 57, 125-139.

WASER, P. M., \& WILEY, R. H. Mechanisms and evolution of spacing in animals. In P. Marler \& J. G. Vandenbergh (Eds.), Handbook of behavioral neurobiology. New York: Plenum, 1980.

(Accepted for publication December 5, 1981.) 